\title{
Imaging Microfluidic Models of the Body
}

\author{
Shuichi Takayama* \\ * Department of Biomedical Engineering and Macromolecular Science \& Engineering Center, \\ University of Michigan, Ann Arbor, MI 48109-2099
}

Many biological studies, drug screening methods, and cellular therapies require culture and manipulation of living cells outside of their natural environment in the body. The gap between the cellular microenvironment in vivo and in vitro, however, poses challenges for obtaining physiologically relevant responses from cells used in basic biological studies or drug screens and for drawing out the maximum functional potential from cells used therapeutically. One of the reasons for this gap is because the fluidic environment of mammalian cells in vivo is microscale and dynamic whereas typical in vitro cultures are macroscopic and static. This presentation will give an overview of efforts in our laboratory to image microfluidic systems that enable spatio-temporal control of both the chemical and fluid mechanical environment of cells. The technologies and methods close the physiology gap to provide biological information otherwise unobtainable. Specific biomedical topics that will be discussed include, imaging of air-liquid two-phase flow and applications to microfluidic tissue engineering of small airway injuries, imaging of intracellular signals in response to subcellular stimulation, imaging of intracellular signals in response to temporally patterned microfluidic stimulations, and imaging single molecules of DNA using tunable nanofluidic systems.

There are many small tubes and channels in the body. Thus, intuitively, one can appreciate that the typical macroscopic culture dish is very different than the environment in the body and cells may "feel" and behave differently. What are the effects that cells feel in the body in their microfluidic environments? The small volumes of fluid surrounding cells would lead to rapid depletion and accumulation of any chemicals cells consume or secrete. Fluid flow in channels would also exert fluid mechanical forces on cells. These conditions are not always well reproduced in macroscopic culture dishes. Polymer microchannel devices, on the other hand, can be constructed to better mimic physiological channel dimensions as well as fluid flow conditions [1]. Autocrine effects can be enhanced, chemical gradients can be created that are more physiological, and fluid mechanical stimulation of the oviduct [2], small airways [3, 4], or blood vessels [5] can be reproduced. This can lead to enhanced pregnancy rates [2], better understanding of lung injury [3, 4], and discovery of new targets for preventing cancer metastasis [5]. Another important consideration for the cell culture microenvironment is three-dimensionality. Microfluidic devices can also be useful in engineering multicellular 3D culture environments. One of the few methods to create purely cellbased 3D cell constructs that allows study of direct cell interactions with minimal exogenous materials is the formation of multicellular aggregates, called spheroids. Microfluidic cell patterning is unique in that $3 \mathrm{D}$ co-culture spheroids can be constructed where initial positions of different cell types can be controlled [6]. This capability led to new insight that the initial position of the cells can dictate the final organization of the cell constructs [6]. The importance of this is seen by the patterned differentiation of ES cells which may be utilized for tissue engineering purposes or used as a platform to study embryonic development [6]. 
Although microfluidics can thus provide beneficial microenvironments for cells, obtaining information of cellular responses under such conditions requires imaging. Imaging of the flow itself is also important to confirm the types of flows that exist inside the devices. In this presentation I will introduce the types of imaging methods we have used successfully with microfluidic cell culture including: phase-contrast optical microscopy [2-6], epi-fluorescence microscopy [3, 5, 6], confocal microscopy [7], FRET imaging [8], life-time imaging [9]. Some of the challenges include dealing with working distance requirements and microfluidic device thickness, optical property differences including refractive index differences and flatness of device issues, reagent delivery and handling issues, and formulation of culture media for optimal cell viability and imaging.

Reference

[1] Shim, J.; Bersano-Begey, T. F.; Zhu, X.; Tkaczyk, A.; Linderman, J.J.; Takayama, S. "Microand Nanotechnologies for Studying Cellular Function" Curr. Top. Med. Chem.,2003, 3, 687-703.

[2] Heo, Y.; Cabrera, L. M.; Bormann, C. L.; Shah, C. T.; Takayama, S.; Smith, G. D. "Dynamic microfunnel culture enhances embryo development and pregnancy rates" Hum Reprod 2010, online [3] Huh, D; Fujioka, H.; Tung, Y.-C.; Futai, N.; Paine, R.; Grotberg, J. B.; Takayama, S. "Acoustically Detectable Cellular-Level Lung Injury Induced by Fluid Mechanical Stresses in Microfluidic Airway Systems” Proc. Natl. Acad. Sci. U.S.A. 2007, 104, 18886-18891.

[4] Tavana, H.; Kuo, C.-H.; Lee, Q. Y.; Mosadegh, B.; Huh, D.; Christensen, P. J.; Grotberg, J. B.; Takayama, S. "Dynamics of Liquid Plugs of Buffer and Surfactant Solutions in a Micro-Engineered Pulmonary Airway Model” Langmuir 2010, online

[5] Song, J. W.; Cavnar, S. P.; Walker, A. C.; Luker, K. E.; Gupta, M.; Tung, Y.-C.; Luker, G. D.; Takayama, S. "Microfluidic Endothelium for Studying the Intravascular Adhesion of Metastatic Breast Cancer Cells" PLoS One 2009, 4, e5756.

[6] Torisawa, Y.; Mosadegh, B.; Luker, G. D.; Morell, M.; O’Shea, K. S.; Takayama, S. "Microfluidic Hydrodynamic Cellular Patterning for Spheroid Co-culture" Integr. Biol., 2009, 1, 649-654.

[7] Huh, D; Tung, Y-C; Wei, H.-H.Grotberg, J.B.; Skerlos, S.J.; Kurabayashi, K.; Takayama, S. "Use of Air-Liquid Two-Phase Flow in Hydrophobic Microfluidic Channels for Disposable Flow Cytometers" Biomed. Microdev. 2002, 4, 141-149.

[8] Sawano, A.; Takayama, S.; Matsuda, M.; Miyawaki, A. "Lateral Propagation of EGF Signaling after Local Stimulation Is Dependent on Receptor Density" Dev. Cell, 2002, 3, 245-257.

[9] Sud, D.; Mehta, G.; Mehta, K.; Linderman, J. J.; Takayama, S.; Mycek, M.-A. "Optical Imaging in Microfluidic Bioreactors Enable Oxygen Monitoring for Continuous Cell Culture" J. Biomed. Opt. 2006, 11, 050504

[10] I thank the many colleagues and funding agencies who have made this research possible. Many of them are acknowledged in the articles referenced. 